Man and Nature

L'homme et la nature

\title{
Rousseau Pédagogue Avant Emile : Le Problème de L’Autorité
}

\section{Jean Terrasse}

Volume 10, 1991

URI : https://id.erudit.org/iderudit/1012635ar

DOI : https://doi.org/10.7202/1012635ar

Aller au sommaire du numéro

Éditeur(s)

Canadian Society for Eighteenth-Century Studies / Société canadienne d'étude du dix-huitième siècle

ISSN

0824-3298 (imprimé)

1927-8810 (numérique)

Découvrir la revue

Citer cet article

Terrasse, J. (1991). Rousseau Pédagogue Avant Emile : Le Problème de L'Autorité. Man and Nature / L'homme et la nature, 10, 199-208.

https://doi.org/10.7202/1012635ar

Copyright (C Canadian Society for Eighteenth-Century Studies / Sociéte canadienne d'étude du dix-huitième siècle, 1991
Ce document est protégé par la loi sur le droit d'auteur. L'utilisation des services d'Érudit (y compris la reproduction) est assujettie à sa politique d'utilisation que vous pouvez consulter en ligne.

https://apropos.erudit.org/fr/usagers/politique-dutilisation/ 


\section{Rousseau Pédagogue Avant Emile: Le Problème de L'Autorité}

Comment expliquer l'attention portée par Rousseau aux problèmes d'éducation? Poser cette question revient à s'interroger sur l'origine de sa vocation d'écrivain. ${ }^{1}$ Ecrire et enseigner sont les deux moyens que le jeune protégé de Mme de Warens envisage pour se faire connaître, parallèlement à une carrière de musicien. Ecrire plutôt qu'enseigner, car Jean-Jacques écrit pour compenser son incompétence comme pédagogue, son ineptie dans le domaine de l'agir.

En 1735, il informe son père de son projet de 'trouver un emploi de Secretaire chez quelque grand Seigneur,' ou de 'servir de Gouverneur a des jeunes gens de qualité,' et il justifie le second choix par 'un peu de talent' qu'il a 'pour l'écriture,' c'est-à-dire pour le 'stile. ${ }^{2}$ En fait, Rousseau semble emprunter un itinéraire courant. Harvey Chisick a montré qu'au XVIIIe siècle, le préceptorat servait souvent de tremplin à une carrière d'écrivain et d'artiste; tout comme la prêtrise, l'enseignement pouvait être un expédient. ${ }^{3}$ Pour le futur auteur d'Emile - de nombreux textes le soulignent - il apparaissait aussi peu fait pour être gouverneur que père de famille. C'est pourquoi le besoin d'enseigner devait, chez lui, se changer en besoin d'écrire.

Le chemin où il s'engage est certes parsemé d'embûches. Durant le demi-siècle précédant la chute de l'Ancien Régime, la situation paradoxale du tiers-état ne transparaît jamais mieux qu'à travers la condition du précepteur; l'oeuvre de Rousseau en témoigne aussi bien que celle de Goethe. Quelque fier qu'il soit de son savoir, le précepteur reste un subalterne. Cette réalité saute aux yeux quand Jean-Jacques se voit contraint de flagorner afin d'obtenir le paiement de ses gages. Comme l'atteste une lettre de 1734, il doit ramper devant Mme de Challes pour la convaincre de lui verser le prix de ses leçons: la damen'avait pas hésité à lui fermer plusieurs fois sa porte alors qu'il venait lui réclamer son dû. ${ }^{4}$ Jusqu'au moment de devenir célèbre, Rousseau a sans doute connu souvent ce genre d'humiliation. Lorsqu'il entre en 1740 au service de Jean Bonnot, seigneur de Mably et grand-prévôt de Lyon, nouvelle déconvenue causée par la modicité de son salaire; mais c'est à Mme de 
Warens qu'il en fait part, tandis qu'il accepte avec empressement les conditions imposées par son patron. ${ }^{5}$

A ce stade, le 'pacte pédagogique' dont fait état Henri Coulet passe par-dessus la tête de l'enfant, puisqu'il est conclu entre le père et le gouverneur. ${ }^{6} C^{\prime}$ est aussi un pacte inégalitaire, dont l'une des parties a le pouvoir de dicter à l'autre les clauses essentielles. En souscrivant aux termes du contrat de service, le gouverneur rêve néanmoins de le transformer en pacte librement consenti; l'idéal est pour lui d'établir avec ses supérieurs une relation de type familial. Dans la négociation qui s'amorce, la compétence du précepteur devrait servir de monnaie d'échange: le savoir contre la considération et la rémunération en argent. Mais Rousseau ne semble pas tirer le meilleur parti de la situation. Dans la lettre à son père, il prétendait en savoir assez que pour instruire 'un jeune gentilhomme'; ${ }^{\prime}$ or le ton change complètement à l'instant où il s'adresse à M. de Mably. Loin de chercher à impressionner, Rousseau tente de s'excuser; il n'a plus rien à offrir qu' un zèle et des soins' dont il espère qu'ils compenseront 'la médiocrité de (s)es lumiéres et de (s)on savoir,' de l' attachement' à défaut d'habileté.

Fausse modestie? C'est peu probable. Face à M. de Mably, Jean-Jacques est obsédé par la crainte de laisser échapper quelque maladresse:

Que s'il m'arrive de commêtre quelque faute, lui écrit-il, je répons d'avance qu'elles seront toujours dignes de pardon, parce qu'elles ne seront jamais volontaires, ce qui n'empêchera pas, Monsieur, que vous n'aiez la bonté de me les faire connoître afin que j'aprenne a les éviter. ${ }^{8}$

Irons-nous jusqu'à supposer que Jean-Jacques espère commettre une faute pour avoir le plaisir d'être pardonné? Une fois introduit chez M. de Mably, il s'arrangera pour forcer le destin. D'abord en tombant amoureux de la maîtresse de maison; puis en dégustant en cachette quelques bouteilles soustraites à la cave familiale. La première faute restera sans conséquence, puisque Rousseau ne poussera pas l'audace jusqu'à déclarer ses sentiments à Mme de Mably; il ne sera coupablequ'à ses propres yeux. ${ }^{9} \mathrm{Al}$ 'égard de la seconde faute, M. de Mably fera preuve de toute l' 'indulgence' souhaitée, et comme on pouvait s'y attendre, ce procédé généreux augmentera l'attachement de Jean-Jacques pour son protecteur. $^{10}$

En somme, la situation se résume ainsi: l'enfant, c'est lui, Jean-Jacques, M. de Mably est son nouveau père. Aucun doute possible, après l'engagement pris par Jean-Jacques qui l'a formulé en toutes lettres: ' Je tâcherai de mériter que vous veuilliez bien me tenir lieu de pére, comme 
je me propose aussi de remplir a vôtre égard tous les devoirs d'un fils respectueux ....' ${ }^{11}$

La lettre à $\mathrm{M}$. de Mably institue une procédure de résolution des conflits à laquelle Rousseau demeurera fidèle jusqu'à la fin de sa vie et qui est indissociable de sa vocation d'artiste. Comme l'a démontré Pierre-Paul Clément, ${ }^{12}$ Jean-Jacques ressent le besoin d'avoir pour juge un père qui lui remet $d^{\prime}$ avance toutes ses fautes en le délivrant de la responsabilité sociale. L'existence de ce père lui donne l'assurance qu'il ne peut commettre que des péchés véniels dus à sa maladresse ou à son ignorance. Inversement, le jeune précepteur peut être tenté de commettre des fautes pour obtenir la preuve que ce père existe et quel'innocence est un état susceptible de se renouveler. Destinées à lui attirer le pardon, ces fautes tranquillisent le futur écrivain parce qu' elles lui font vivre une situation de dépendance filiale qu'il avait déjà recherchée auprès de Mme de Warens. Inutile de s'appesantir sur les raisons qui l'amènent à reconstituer la famille qui lui a manqué; il n'est pas nécessaire d'être un fanatique de psychanalyse pour trouver dans l'enfance l'explication d'une telle singularité. C'est ici le lieu de se souvenir de l'évocation dramatique que l'auteur des Confessions a laissée de ses années d'apprentissage chez Abel Du Commun. Les insultes, les coups avaient succédé trop vite à la tendresse paternelle. Il n'y aurait pas d'autre alternative que 'la dépendance filiale' que Rousseau s'efforcera de recréer dans ses amitiés et même dans ses amours, et 'l'esclavage servile' dont la société lui a imposé les chaînes et qu'il dénoncera dans ses écrits. ${ }^{13}$ De l'un à l'autre de ces états, le progrès est indéniable. Tel est le trajet effectué depuis que Rousseau a quitté Genève; il va dans le sens $d^{\prime}$ une affirmation de soi, d'un gain de liberté que permettait l'exercice des facultés intellectuelles, par le biais du 'Sistème d'étude' adopté depuis le premier séjour aux Charmettes. Chez M. de Mably, Jean-Jacques continue sur sa lancée.

Pour le précepteur, la difficulté n'est pas seulement d'assumer dignement la condition de travailleur à gages. Un autre problème sérieux est l'existence même de l'élève, avec lequel il faut nécessairement composer. Si nous supposons réglées les questions d'amour-propre dont son état lui donne une conscience aiguë, le précepteur n'est pas encore au bout de ses peines, car il doit être fait pour ce métier. La façon dont il voit son élève est liée à sa propre compétence à enseigner, par conséquent à l'ascendant qu'il est à même d'exercer sur son disciple. C'est ainsi que le problème de l'autorité ne peut se comprendre qu'en fonction de l'habileté personnelle du gouverneur, de son 'professionnalisme': l'autorité 'fait problème' quand la vocation est prise en défaut.

Dans sa lettre à Mme de Warens du ler mai 1740, lettre où il lui détaille les conditions de son engagement chez M. de Mably, Rousseau a failli 
oublier l'existence de son élève. Il se rattrape in extremis, mais sans parvenir à cacher son inquiétude:

Quant à mon petit élève, on ne sauroit lui refuser d'être très aimable, mais je ne saurois encore vous dire s'il aura le coeur également bon, parce que souvent ce qui paroit à cet âge des signes de méchanceté, $\mathrm{n}^{\prime}(\mathrm{en})$ sont en effet que de vivacité et d'étourderie. ${ }^{14}$

En fait de 'petit élève,' il y en avait deux: Sainte-Marie, qui n'avait pas six ans, et Condillac, qui n' en avait pas quatre. Tous deux inéducables, à en croire les Confessions: l'aîné, 'l'esprit assez ouvert, assez vif, étourdi, badin, malin, mais d'une malignité gaye'; le cadet, 'presque stupide, musard, têtu comme une mule, et ne pouvant rien apprendre.' Rousseau emploie avec eux une méthode surprenante: 'le sentiment, le raisonnement, la colère.' Il lui arrive de pleurer avec Sainte-Marie; encore moins capable de contrôler Condillac, il entre en fureur contre lui. ${ }^{15}$ Le bilan est facile à dresser: échec sur toute la ligne; le précepteur est entièrement débordé par ses élèves. Raison de plus pour tenter d'amadouer le père, assurément plus raisonnable que ses deux fils, pour lui faire étalage de son esprit, lui exposer ses idées. C'est probablement ce qui incite Rousseau à écrire le Mémoire présentéa à Monsieur de Mably sur l'éducation de M. son fils.

L'ouvrage mène de front l'exposé d'un programme d'éducation et le plaidoyer pro domo. Selon toute apparence, l'avenir des deux enfants préoccupait moins leur précepteur que ses relations avec M. de Mably. Démuni devant le présent, Rousseau veut rassurer sur ses entreprises futures. Et il comprend qu'il ne peut établir mieux sa crédibilité qu'en la fondant sur son incompétence, du moment qu'il est incapable de se changer.

Un exemple: vingt ans avant la publication d'Emile, Jean-Jacques affirme déjà que le but de l'éducation est de rendre l'enfant heureux, mais comment enseignera-t-il ce qu'il ' $n$ 'a pu trouver pour (lui)même' $?^{16}$ La réponse n'est pas ailleurs que dans l'énoncé de la question: on enseigne ce qu'on n'a pu mettre en pratique en le transformant en littérature. Tel est le paradoxe fondamental autorisant la confusion entre l'enseignement et l'écriture: ce qu' on est empêché de faire, on l'écrit-on écrit pour cette seule raison qu'on ne peut agir. Plus tard, Rousseau enfermera de même l'action politique dans le dire de l'écrivain ou dans les tables de la loi. Nous lisons tout au début du Contrat social:

On me demandera si je suis prince ou législateur pour écrire sur la Politique? Je réponds que non, et quec'est pour cela que j'écris sur la Politique. Si j'étois prince 
ou législateur, je ne perdrois pas mon tems à dire ce qu'il faut faire; je le ferois, ou je me tairois. ${ }^{17}$

Le problème de l'autorité se pose à peu près dans les mêmes termes. L'ascendant que Jean-Jacques n'a pu acquérir sur ses élèves, c'est maintenant au texte de le fonder. A défaut de capter l'attention des enfants, Rousseau veut retenir celle du père. Ce faisant, il transforme la nature de l'autorité, confondue avec la fascination de la lecture. En devenant écrivain, le gouverneur gagne le droit de parler de lui-même. Comme éducateur, il était normalement tenu de dissimuler ses faiblesses; en s'adressant à $\mathrm{M}$. de Mably, il peut cesser de lutter contre lui-même, et tirer parti de ses défauts.

Pour faire son apologie, Rousseau en dresse la liste, mais en changeant le signe 'moins' en signe 'plus': alors les carences deviennent des qualités. Procédé irritant, qui vaudra plus tard à l'écrivain la réputation de séduire par des sophismes. Mais l'impression de cynisme laissée par certains de ses raisonnements tient à l'origine même de son écriture, liée au manque, à la privation, à l'absence, suivant la thèse célèbre de Derrida. Dans le Mémoire à $M$. de Mably, Rousseau a déjà une façon inimitable de dire qu'il a ce qu'il n'a pas et qu'il n'a pas ce qu'il a. Ainsi, la timidité dont il se plaint d'être affligé devrait disqualifier Jean-Jacques comme précepteur ou du moins l'empêcher d'aborder avec M. de Mably la question de l'autorité: comment un homme timide peut-il prétendre se faire obéir? C'est pourtant l'inverse qui se produit. Cette timidité accroît le prestige du gouverneur, à ses propres yeux en tout cas, parce qu' elle 'est rarement le défaut des sots' et que d'en souffrir lui permet de se croire mieux placé qu'un autre pour corriger ce défaut chez son élève. ${ }^{18}$

De même, Rousseau encouragera Sainte-Marie à fréquenter le monde, avec d'autant plus de conviction que lui-même y a toujours fait piètre figure. Il est amusant de se souvenir que Mme de Mably a vainement tenté de guérir Jean-Jacques de ses gaucheries. Lui-même le raconte dans les Confessions:

$\mathrm{J}^{\prime}$ avois été recommandé par $\mathrm{Mad}^{\mathrm{e}}$ Deybens à Mad ${ }^{\mathrm{e}}$ de Mably. Elle l'avoit priée de former mes maniéres et de me donner le ton du monde; elle y prit quelques soins et voulut que j'apprisse à faire les honneurs de sa maison; mais je m'y pris si gauchement, j'étois si honteux, si sot qu'elle se rebuta et me planta la. ${ }^{19}$

Cette mauvaise expérience n'empêche pas Rousseau d'insister, dans le Mémoire, sur l'importance de l'éducation mondaine. Au contraire, elle met en valeur sa bonne foi. Vous ne pouvez, assure-t-il à son inter- 
locuteur, me soupçonner d'agir par intérêt, car 'bien loin d'être taxé d'aimer le monde et la dissipation, je sais, $M .$, qu' on a cherché plus d'une fois à me faire passer auprès de vous pour un caractére triste et misanthrope ..... ${ }^{20}$ Le maître est donc pour l'élève un exemple à ne pas suivre, et $c^{\prime}$ est pourquoi il doit être le maître. Ce paradoxe s'impose à tout lecteur attentif aux subtilités du texte, par le truchement d'un raisonnement en quatre points:

1. Pris par certains 'pour un esprit sauvage et pédant qui hors d'êtat de connoître le monde $l^{\prime}$ (est) encore plus d'y former un jeune Elêve,' Rousseau admet la vraisemblance de ces 'accusations.'

2. Cependant, il découvre 'avec joie qu'elles n'ont pas produit beaucoup d'effet' sur M. de Mably, et il se promet de faire les 'efforts' nécessaires pour qu'elles lui paraissent 'tous les jours plus injustes.'

3. Selon son aveu, trois obstacles risquent de lui rendre la tâche ardue: 'un penchant invincible à la mélancolie,' 'une timidité insurmontable,' enfin 'une profonde indifférence pour tout ce qu'on appelle brillant.'

4. Ces insuffisances sont contrebalancées par une 'douceur de caractère' tout à l'opposé de l'humeur que certaines personnes lui attribuent. Selon Rousseau, M. de Mably peut trouver une preuve de cette heureuse disposition dans le 'plaisir' que son fils aîné 'marque' à vivre aux côtés de son gouverneur. ${ }^{21}$ L'élève déciderait en dernier ressort de ce que $\mathrm{M}$. de Mably doit penser du précepteur: telle est la situation que la rhétorique de l'oeuvre suggère à l'esprit du lecteur. Derrière elle se cache néanmoins une réalité bien différente, car le Mémoire montre aussi que Jean-Jacques compte sur M. de Mably pour contraindre l'enfant à l'obéissance.

Outre sa maladresse naturelle, la condition subalterne du précepteur l'oblige, en effet, à se tourner vers le père pour se faire écouter des enfants. Comme M. de Mably peut seul le revêtir de l'autorité nécessaire, Jean-Jacques propose un pacte par lequel son protecteur lui délèguera ses pouvoirs: l'idée sera reprise dans Emile où elle jouera le même rôle essentiel. Précision intéressante, l'enfant doit être informé du pacte; le père lui adressera à cette occasion un 'petit discours' suivi d'une déclaration par laquelle il rendra le gouverneur 'dépositaire' de son 'autorité.' Afin de rassurer son interlocuteur, Rousseau affirme que la cérémonie n'a d'autre but que de faire impression sur l'enfant et que lui-même n'usera de son crédit auprès de Sainte-Marie que pour l'amener à se 
conformer aux volontés de son père. ${ }^{22}$ En somme, Jean-Jacques veut que M. de Mably lui donne pouvoir de rendre son élève docile aux instructions que $\mathrm{M}$. de Mably prescrit à Jean-Jacques de lui faire suivre. Le procédé insère l'écrivain ou le pédagogue dans un circuit de relations familiales dont il attend qu'elles le protègent contre lui-même et contre la société. Désormais, le gouverneur commandera à son élève comme un père commande à son fils, mais ce faisant, il obéira à $\mathrm{M}$. de Mably comme un fils obéit à son père. L'auteur du Mémoire élimine ainsi le risque inhérent à toute action qui engage l'individu envers la société; pédagogue, il forme un enfant en se déchargeant sur un autre de ses responsabilités majeures: l'écriture permet bien des tours de passepasse.

En plus de la clause de la passation des pouvoirs, le pacte pédagogique contient des articles qui règlementent l'exercice de l'autorité que le père a déléguée temporairement au précepteur. Suivant ces dispositions, le père ne fera de cadeau à l'enfant qu'après s'être assuré que le gouverneur est content de sa conduite; l'élève se verra refuser toute espèce de grâce qu'il n'aura demandée 'par la bouche de son Maître ..... ${ }^{, 23}$ A vrai dire, les interventions de M. de Mably ne se limitent pas aux situations mettant en jeu le principe d'autorité; elles peuvent donner prise directement sur l'éducation intellectuelle et morale. A l'occasion, M. de Mably jouera le rôle de répétiteur; ${ }^{24}$ il prendra des mesures pour corriger Sainte-Marie de sa timidité excessive; ${ }^{25}$ Jean-Jacques recourra également à son assistance pour détourner le fils aîné des amusements frivoles. ${ }^{26}$ Bref, le rôle dévolu au père estsi important que le précepteur craint l'objection de s'être réservé la meilleure part: ' vous n'avez pas lieu de vous appercevoir jusqu'à présent que je cherche à fuir le travail,' observe-t-il à l'adresse de M. de Mably, en se défendant de vouloir changer un métier ingrat en une 'occupation... agréable. ${ }^{27} \mathrm{Il}$ reste que le Mémoire le montre plus préoccupé d'instruire le père que d'éduquer les enfants; si la réussite du pédagogue semble problématique, séduire le lecteur est du moins une entreprise à la portée d'un grand écrivain.

Rousseau quittera M. de Mably, comme il le dit dans les Confessions, 'dégouté d'un métier auquel je n'étois pas propre.... ${ }^{28} \mathrm{Il}$ est piquant de noter qu'en 1743 , moins d'un an après son arrivée à Paris, il trouve l'occasion de réutiliser le Mémoire dans des circonstances analogues à celles qu'il a connues à Lyon. Résumons les faits. Rousseau s'éprend de Mme Dupin, qu'il a aperçue à demi-nue à sa toilette. 'Timidité naturelle,' crainte d'être chassé d'une 'maison opulente,' il n'ose parler, mais cette fois, se hasarde à écrire. Mme Dupin lui fait de vive voix une remontrance en guise de réponse. Quelque temps après, elle accomplit une démarche qui prouve qu'elle lui a gardé sa confiance: comme Chenon- 
ceaux, son fils, doit changer de gouverneur, elle demande à Rousseau de 'veiller' sur lui 'pendant' les 'huit ou dix jours' où il restera seul. ${ }^{29}$ Cette requête répond probablement à une lettre que Rousseau a envoyée à Mme Dupin le 9 avril 1743 dans l'espoir de rentrer en grâce, lettre dans laquelle il proposait une sorte de marché: 'Je vous supplie ..., Madame, de pardonner la liberté que j'ai prise de vous écrire cette lettre, en vous adressant un mémoire que vous $m$ 'avez fait l'honneur de me demander; je me réponds assez du motif qui l'a dictée, pour n'en craindre aucune nouvelle disgrâce. ${ }^{30}$

Il s'agit du Mémoire à $M$. de Mably dans sa version apparemment la plus ancienne, la plus riche en aveux personnels. Ne peut-on supposer que Rousseau comptait sur ces aveux pour attendrir Mme Dupin et peut-être pour apaiser son mari? Une fois encore, le Mémoire remplirait une double fonction: répondre aux préoccupations de Mme Dupin au sujet de son fils; obtenir le pardon des fautes dont Jean-Jacques se serait rendu coupable, en renvoyant l'image d'un coeur exempt de malice. Cette fois après coup, le geste associe inconsciemment le travail de l'écriture à l'incompétence de l'amant. Or le récit des Confessions expose le ratage du précepteur succédant aux déconvenues de l'amant:

Je passai ces huit jours dans un supplice que le plaisir d'obéir à $\operatorname{Mad}^{\mathrm{e}}$ Dupin pouvoit seul me rendre souffrable: car le pauvre Chenonceaux avoit dès lors cette mauvaise tête qui a failli deshonorer sa famille, et qui l'a fait mourir à l'lle de Bourbon. Pendant que je fus auprès de lui je l'empéchai de faire mal à lui-même ou à d'autres, et voila tout: encore ne fut-ce pas par une médiocre peine, et je ne m'en serois pas chargé huit autres jours de plus, quand $\mathrm{Mad}^{\mathrm{e}}$ Dupin se seroit donnée à moi pour récompense. ${ }^{31}$

Durant cet intermède, le problème de l'autorité a été la pierre d'achoppement, comme auparavant chez M. de Mably. Dès lors, il n'est pas étonnant que les idées de Wolmar en matière d'éducation soient centrées sur ce même problème: dans la lettre V, 3 de la Nouvelle Héloïse, nous voyons les enfants de Julie lui obéir au moindre signe, sans que la mère doive jamais recourir à l'intimidation. Je voudrais montrer, si je disposais de plus de temps, comment Emile peut être lu sous le même éclairage. Des générations de lecteurs se sont demandé comment Rousseau avait concilié la rédaction d'un grand traité d'éducation avec $l^{\prime}$ abandon de ses enfants. Plusieurs critiques ont supposé qu'il s'agissait d'une oeuvre d'expiation, bien que Jean-Jacques ait fait des déclarations quelque peu contradictoires à ce sujet. En fait, Emile a été écrit dans des conditions semblables au Mémoire à M. de Mably. Incapable d'élever ses enfants, Rousseau ne pouvait assumer sa paternité que par l'écriture. 
'... C'est moi qui suis le vrai pére d'Emile, c'est moi qui l'ai fait homme,' s'écrie-t-il en confondant sa voix avec celle du précepteur. ${ }^{32}$. $D^{\prime}$ un autre côté, il est absolument sincère quand il déclare qu'un homme qui ne remplit pas ses devoirs de père ne mérite pas sa place dans la société. ${ }^{33}$ Rousseau a besoin du détour de l'écriture pour s'acquitter des obligations que la nature ou la société prescrivent, il apparaît surtout que l'écriture seule lui permet d'enseigner. 'Je suis trop pénétré de la grandeur des devoirs d'un précepteur,' avoue l'auteur d'Emile, 'je sens trop mon incapacité pour accepter jamais un pareil emploi de quelque part qu'il me soit offert .... ${ }^{34}$ L'oeuvre littéraire n'a pas vraiment pour fonction d'activer le remords et de réparer la faute, mais le constat de carence, et peut-être le sentiment de culpabilité qui en résulte, ont rendu l'oeuvre à la fois possible et nécessaire.

\section{JEAN TERRASSE}

Université McGill

\section{Notes}

1 Cette communication résume quelques-unes des thèses d'un ouvrage en préparation sur la rhétorique des écrits pédagogiques de Rousseau.

2 Correspondance complète de Jean-Jacques Rousseau, éd. R.A. Leigh, t. I, n. 11, pp. 30-31.

3 Harvey Chisick, The Limits of Reform in the Enlightenment: Attitudes toward the Education of the Lower Classes in Eighteenth-Century France (Princeton, New Jersey: Princeton University Press, 1981), p. 28.

4 C.C., éd. R.A. Leigh, t. I, n. 8, p. 22.

5 C.C., t. I, n. 37, p. 121.

6 Henri Coulet, 'Le pacte pédagogique dans Emile,' dans Jean-Jacques Rousseau. Quatre études de Jean Starobinski, Jean-Louis Lecercle, Henri Coulet, Marc Eigeldinger (Neuchâtel, La Bâconnière, 1978), p. 76.

7 C.C., t. I, n. 11, p. 31.

8 C.C., t. I, n. 36, p. 120.

9 Confessions, VI, dans O.C., éd. de la Pléiade, t. I, p. 268.

10 Idem, p. 269.

11 C.C., t. I, n. 36, p. 120.

12 Pierre-Paul Clement, Jean-Jacques Rousseau. De l'éros coupable à l'éros glorieux (Neuchâtel, à la Baconnière, 1976), p. 509.

13 Confessions, I, dans O.C., t. I, p. 31.

14 C.C., t. I, n. 38, p. 123.

15 Confessions, VI, dans O.C., t. I, p. 267. 
16 Mémoire à M. de Mably, dans O.C., t. IV, p. 12.

17 Contrat social, I, 1, dans O.C., t. III, p. 351.

18 Mémoire à M. de Mably, dans O.C., t. III, p. 351.

19 Confessions, VI, dans O.C., t. I, p. 268.

20 Mémoire à M. de Mably, dans O.C., t. IV, p. 21.

21 Idem, p. 21-22.

22 Idem, p. 6.

23 Idem, p. 5.

24 Idem, p. 28.

25 Idem, p. 23.

26 Idem, p. 26.

27 Idem, p. 10.

28 Confessions, VI, dans O.C., t. I, p. 269.

29 Confessions, VII, dans O.C., t. I, pp. 291-93.

30 C.C., éd. R.A. Leigh, t. I, n. 52, p. 182.

31 Confessions, VII, dans O.C., t. I, p. 293.

32 Emile, V, dans O.C., t. IV, p. 765.

33 Emile, I, dans O.C., t. IV, pp. 262-63.

34 Idem, p.264. 